\title{
Excess of Proton Mean Kinetic Energy in Supercooled Water
}

\author{
A. Pietropaolo, R. Senesi, and C. Andreani \\ Dipartimento di Fisica and Centro NAST (Nanoscienze \& Nanotecnologie \& Strumentazione), \\ Università degli Studi di Roma “Tor Vergata," Via della Ricerca Scientifica 1, 00133 Roma, Italy \\ A. Botti, M. A. Ricci, and F. Bruni* \\ Dipartimento di Fisica "E. Amaldi," Università degli Studi di Roma Tre, Via della Vasca Navale 84, 00146 Roma, Italy
} (Received 3 October 2007; published 27 March 2008)

\begin{abstract}
We find, by means of a deep inelastic neutron scattering experiment, a significant excess of proton mean kinetic energy $\left\langle E_{k}\right\rangle$ in supercooled water, compared with that measured in stable liquid and solid phases. The measured values of $\left\langle E_{k}\right\rangle$ at moderate degrees of supercooling do not fit the predicted linear increase with temperature observed for the water stable phases. This anomalous behavior is confirmed by the shape of the measured momentum distribution, thus supporting a likely occurrence of ground-state quantum delocalization of a proton between the $\mathrm{O}$ atoms of two neighboring molecules. These results strongly suggest a transition from a single-well to a double-well potential felt by the delocalized proton, with a reduced first neighbor O-O distance, in the supercooled state, as compared to ambient condition.
\end{abstract}

DOI: 10.1103/PhysRevLett.100.127802

PACS numbers: 61.25.Em, 78.70.Nx

The anomalous properties of water have attracted great attention from the scientific community for a long time and are still a topic of intense research. These remarkable properties, most pronounced in the supercooled metastable state [1], can be ascribed to water's unique structure, consisting of a random and fluctuating three-dimensional network of hydrogen bonds [2]. Despite the combined effort of powerful molecular dynamics simulation and novel experimental methods, a complete description of water is still missing. Therefore, this apparently simple liquid still represents a challenging puzzle [3-5]. These issues have received great interest in recent years thanks to possibilities opened by novel experimental techniques, detailed theoretical predictions, and computer simulation methods. In particular, the development of pulsed neutron sources has allowed the remarkable advance of the deep inelastic neutron scattering (DINS) technique [6,7]. DINS is based on measurements at high energy, $\hbar \omega$, and high momentum, $\hbar q$, transfers, thus providing a probe of both the short-time $\left(t \leq 10^{-15} \mathrm{~s}\right)$ dynamics and local $(r \leq 1 \AA)$ environment of the atoms in materials [6,7]. The high energy and momentum transfers achieved allow us to describe the scattering process within the framework of the impulse approximation (IA) [7-9]. The scattering cross section is then expressed in terms of the single particle momentum distribution, $n(p)$, whose variance is related to the mean kinetic energy $\left\langle E_{k}\right\rangle$. In the case of water protons, for instance, the $n(p)$ and $\left\langle E_{k}\right\rangle$ provide a richness of information about the potential surface that the proton experiences, including the effects of hydrogen bonding. This complements microscopic structural studies and allows a direct comparison with quantum Monte Carlo simulations [10,11]. These possibilities make the DINS technique a unique and well established tool to investigate the hydrogen bonding of water under various conditions [12-16].
Here we report on an experimental determination of the proton $n(p)$ and $\left\langle E_{k}\right\rangle$ for a sample of bulk moderately supercooled water. This experiment was performed on a large sample of bulk water, thus avoiding the perturbation introduced by a confining substrate, often used to deeply supercool nanosized water samples, and special care was taken to avoid sample crystallization. DINS experiments have been carried out on the VESUVIO spectrometer [7] at the ISIS spallation neutron source (U.K.). The samples were contained in a disk-shaped aluminum can $(5 \mathrm{~cm}$ diameter, $1 \mathrm{~mm}$ thickness) with inner Teflon coating to prevent ice formation. The occurrence of Bragg's peaks in the thermal neutron energy range was constantly monitored, during data acquisition, to check for freezing. Two temperature sensors, placed in proximity of the sample, monitored heat release due to ice formation. Upon sample crystallization, data collected during the previous two hours were discarded; subsequently the sample was heated to $300 \mathrm{~K}$, and then cooled down. Data have been acquired employing the single difference (SD) and the double difference (DD) methods [17], the latter providing a narrower instrumental resolution (see Fig. 1). Within the IA framework the dynamical structure factor, $S_{I A}(\vec{q}, \omega)$, is related to the $n(p)$ through the relation:

$$
S_{I A}(\vec{q}, \omega)=\int n(\vec{p}) \delta\left(\omega-\frac{\hbar q^{2}}{2 M}-\frac{\vec{p} \cdot \vec{q}}{M}\right) d \vec{p},
$$

where $\frac{\hbar^{2} q^{2}}{2 M}$ is the recoil energy of the struck atom of mass $M$. The dynamical structure factor is then expressed in terms of a neutron Compton profile (NCP) [7]: $F(y)=\frac{\hbar q}{M}$ $S_{I A}(\vec{q}, \omega)$, where $y=\frac{M}{\hbar q}\left(\omega-\frac{\hbar q^{2}}{2 M}\right)$ is the West scaling variable [7]. The NCP line shape is convolved with the instrumental resolution function and is represented as a series expansion in Hermite polynomials: 


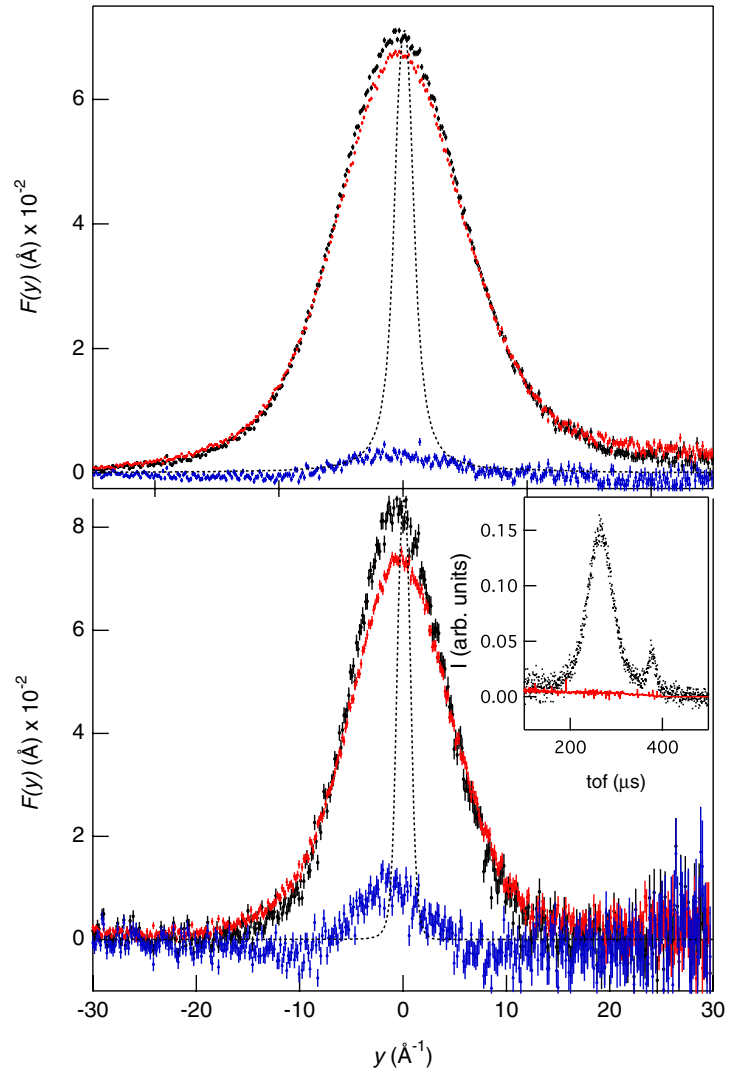

FIG. 1 (color online). Neutron Compton profile, $F(y)$, averaged over the 32 detectors, of supercooled water at $269 \mathrm{~K}$ (red symbols) and $271 \mathrm{~K}$ (black symbols): single difference method (upper panel), double difference method (lower panel). Experimental spectrometer resolutions in $y$-space are shown as dashed lines. Blue symbols (or data set with maximum amplitude at $y=$ 0 equal to 0.03 and 0.13 , respectively) in upper and lower panels represent the NCP difference between the two temperatures. Values for $\sigma$ and the non-Gaussian coefficient $c_{2}$ are $5.66 \pm$ $0.03 \AA^{-1}$ and $0.378 \pm 0.011$ at $269 \mathrm{~K} ; 6.05 \pm 0.03 \AA^{-1}$ and $0.443 \pm 0.008$ at $271 \mathrm{~K} . c_{1}$ is set to zero by definition, and $c_{n \geq 3}$ are found to be negligible. The inset shows an example of fixedangle raw time-of-flight data (black dots) collected by the detector at $38^{\circ}$ at $269 \mathrm{~K}$, together with the multiple scattering contribution (red line) evaluated by Monte Carlo simulation [7].

$$
F(y)=\frac{e^{-y^{2} / 2 \sigma^{2}}}{\sqrt{2 \pi} \sigma}\left[1+\sum_{n} \frac{c_{n}}{2^{2 n} n !} H_{2 n}\left(\frac{y}{\sqrt{2} \sigma}\right)\right]
$$

The coefficients $c_{n}$ and $\sigma$, appearing in the series expansion, were determined by a least squares fitting procedure, and small corrections due to deviations from the IA were taken into account [7]. We did not attempt to correct for the so-called intensity deficit seen for hydrogen relative to heavier nuclei [18-20]. Although the issue of the intensity deficit in the DINS experiment is still open to debate, it should be noted that in systems where the $n(p)$ and the NCP are purely Gaussian, such as $\mathrm{ZrH}_{2}$, no distortion of the measured NCP is indeed observed [21]. The $n(p)$ is expressed by the expansion [7]

$$
n(p)=\frac{e^{-p^{2} / 2 \sigma^{2}}}{(\sqrt{2 \pi} \sigma)^{3}} \sum_{n} c_{n}(-1)^{n} L_{n}^{1 / 2}\left(\frac{p^{2}}{2 \sigma^{2}}\right)
$$

where $L_{n}^{1 / 2}$ are generalized Laguerre polynomials. Values for $\sigma$ and the non-Gaussian coefficient $c_{2}$ are given in the caption of Fig. 1. A sensible difference between the NCP data set collected at the two temperatures is clearly visible in both (SD and DD) experimental configurations (see Fig. 1).

Figure 2 shows $\left\langle E_{k}\right\rangle$ as a function of temperature. In the supercooled states there is an excess of $\left\langle E_{k}\right\rangle$ of about $7 \mathrm{~kJ} / \mathrm{mol}(\sim 50 \mathrm{meV})$ compared to ambient temperature. This is very close to the H-bond energy. Importantly, a small change of sample temperature, from $269 \mathrm{~K}$ to $271 \mathrm{~K}$, causes a $10 \%$ increase of $\left\langle E_{k}\right\rangle$. Such a remarkable change was already visible in the raw data, as peak intensities and tails show a clear difference between the two data sets (blue symbols, with maximum amplitude at $y=0$ equal to 0.03 and 0.13 in the upper and lower panels of Fig. 1, respectively). In addition, the same experimental setup and data analysis procedure described above have been used for room temperature water DINS experiments. Therefore observed differences in the NCP and $\left\langle E_{k}\right\rangle$ are to be ascribed to the different thermodynamic state investigated in the present study.

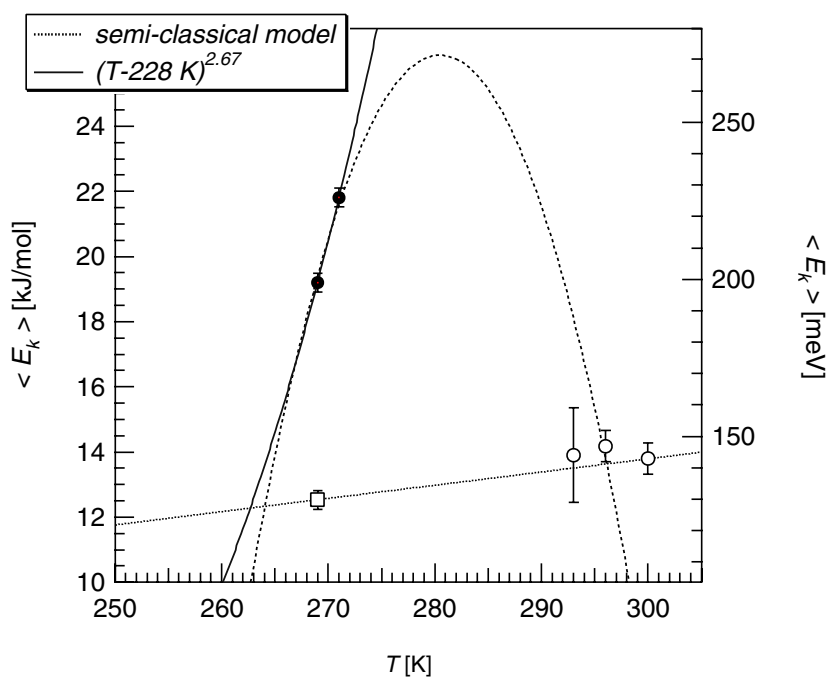

FIG. 2 (color online). Proton mean kinetic energy $\left\langle E_{k}\right\rangle$ measured with DINS experiments on water samples under different thermodynamic conditions as a function of temperature. Supercooled phase $\bullet$, present results, stable liquid phase ○ data taken from Refs. [13,14]; ice $\square$, data taken from Ref. [13]. MCT temperature dependence of proton mean kinetic energy, $\left\langle E_{k}\right\rangle \sim\left(T-T_{s}\right)^{\gamma}$, is shown as a solid line, with $T_{s}$ set equal to $228 \mathrm{~K}$ and $\gamma$ estimated equal to 2.67. Linear temperature dependence of $\left\langle E_{k}\right\rangle$ (see text) is shown as a dotted line. Polynomial fit of mean kinetic energies for supercooled and liquid phases of water is shown as a dashed line. 
This dramatic temperature dependence of $\left\langle E_{k}\right\rangle$ can be, at least qualitatively, described by a power law function $\left\langle E_{k}\right\rangle \sim\left(T-T_{s}\right)^{\gamma}$ (Fig. 2, solid line) consistent with the mode coupling theory (MCT) predictions [22]. Given the limited number of data points available, the singular temperature $T_{s}$ has been fixed to $228 \mathrm{~K}$ [23], while the exponent $\gamma$ has been estimated equal to 2.67, in good agreement with values found for the description of the divergence of water structural and dynamical properties at $T_{s}$ [24].

To extend the comparison between the $\left\langle E_{k}\right\rangle$ values measured for supercooled metastable water and those obtained for water stable phases, data available for bulk water at $293 \mathrm{~K}$ [14], $296 \mathrm{~K}$ [13], and $300 \mathrm{~K}$ [25] have been used. The temperature dependence of $\left\langle E_{k}\right\rangle$ in these stable phases, shown in Fig. 2 as a dotted line, has been derived taking into account translational, rotational, and vibrational contributions [7,12], also making use of the optical spectroscopic data available in the literature. In particular, the translational and vibrational contributions have been treated semiclassically, while a full quantum description has been adopted for the vibrational contribution. This approach resulted in a linear temperature dependence of $\left\langle E_{k}\right\rangle$, which fits not only its values for water from ambient to supercritical states, but the $\left\langle E_{k}\right\rangle$ value for bulk ice at $269 \mathrm{~K}$ [13] as well (Fig. 2). As previously noted, the $\left\langle E_{k}\right\rangle$ values measured for supercooled water at $T=269$ and $271 \mathrm{~K}$ are largely in excess of the above predictions. This significant deviation should have a clear signature in the shape of the $n(p)$. To highlight the differences between the $n(p)$ measured in the supercooled states and those measured in the crystalline and liquid stable phases [13], we have plotted the radial momentum distribution $4 \pi p^{2} n(p)$

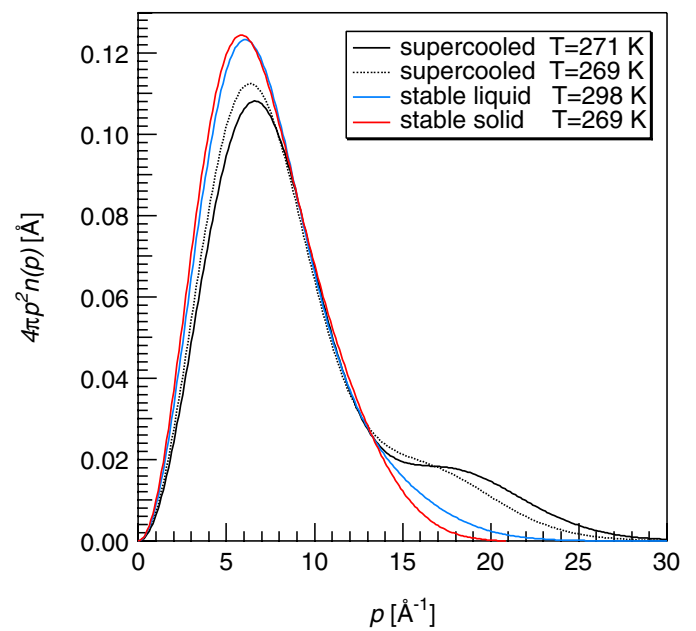

FIG. 3 (color online). Radial proton momentum distribution, $4 \pi p^{2} n(p)$, for water under different thermodynamic conditions as a function of $p$. Supercooled water at $271 \mathrm{~K}$ (solid line), supercooled water at $269 \mathrm{~K}$ (dotted line), stable liquid phase at $298 \mathrm{~K}$ (blue line), stable solid at $269 \mathrm{~K}$ (red line). Experimental uncertainties are less than $\pm 1 \%$. in Fig. 3. As anticipated, the $4 \pi p^{2} n(p)$ measured in two supercooled states shows a low-momentum narrowing along with a shoulder at about $p=17 \AA^{-1}$, while no such feature is visible for the two stable phases. It has been shown that secondary peaks in the radial momentum distribution indicate quantum delocalization, or coherent interference of the proton over the two sites of a doublewell potential $[15,26,27]$. In other words, assuming that each water proton is shared by a covalent bound oxygen and a H-bonded one, the proton will be coherent over two separated sites along the H-bond direction. The similarity between the H-bond and the proton mean kinetic energies, indeed, suggests that each water proton can easily break and reform hydrogen bonds with one of his two first neighboring oxygens. The presence of the shoulder in the $4 \pi p^{2} n(p)$ measured in the supercooled states (solid and dotted lines, Fig. 3), along with its absence for water at ambient condition and for the ice sample (blue and red lines, Fig. 3), does confirm the quantum nature of the excess of mean kinetic energy of water protons in the metastable state. The differences shown in Fig. 3 are also a strong indication of the occurrence of a modification of the potential experienced by water protons. The latter is compatible with the transition from a single well for the stable water phases [13] to a double-well potential for supercooled water, although the isotropic sample, providing a spherically averaged $n(p)$, does not allow one to distinguish between a symmetric vs an asymmetric shape of this latter potential. The resulting delocalization of the proton between the oxygen atoms of two neighboring water molecules is at least consistent with the finding that a proton can become strongly delocalized between two oxygen atoms if the average $\mathrm{O}-\mathrm{O}$ distance is $<2.8 \AA$, leading to a very broad double-well potential in the energy region, corresponding to the second excited state of the O-H stretch vibration [28]. Although these results are not directly comparable to our observations, as these deal with a ground-state property of the proton, it should be noted that the distance between the two minima of the potential obtained from the fitting of the pump-probe transient spectra [28] coincides with the distance of coherence given by the radial $n(p)$ (Fig. 3). Recalling that the relation $p=2 \pi / r$ holds, we can estimate the distance $r$ between the two peaks of the radial $n(p)$ for supercooled water, as equal to $0.6 \AA$, in very good agreement with the reported value [28]. The first neighbor oxygen-oxygen distance is clearly dictating the shape of the potential felt by the water proton, even for the ground state examined in this Letter. This observation led us to discuss and interpret the excess of $\left\langle E_{k}\right\rangle$ in supercooled water (Fig. 2) in terms of the average oxygen-oxygen distance between two water molecules in the supercooled and in the stable liquid phases. To this end, we have further analyzed recent neutron diffraction data on supercooled bulk water [29]. Figure 4 shows the O-O radial distribution function: a clear 


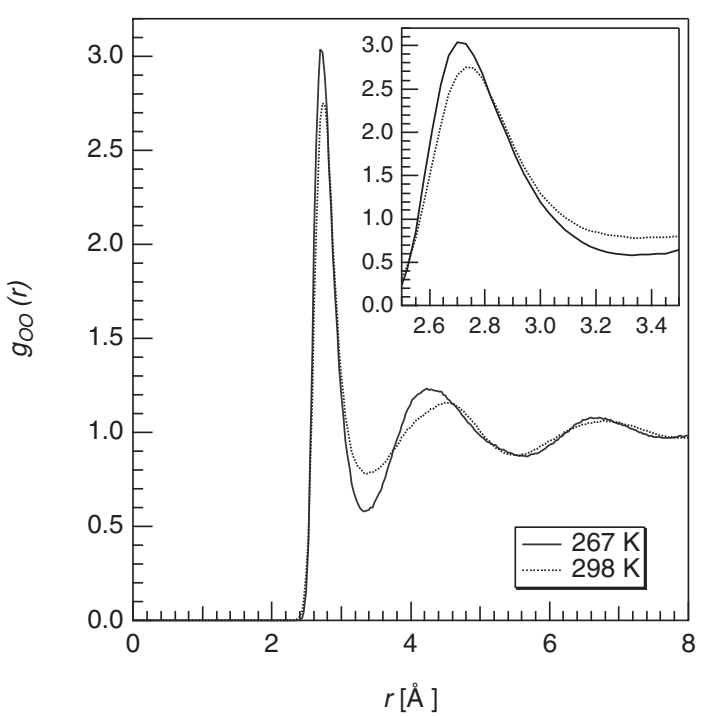

FIG. 4. Oxygen-oxygen radial distribution function for ambient (dotted line) and supercooled (solid line) water [29]. The inset shows a magnification of the first neighbor shell region.

shift to smaller values of all the peak positions, indicating the most likely intermolecular distances between water molecules away from a central one, is evident comparing supercooled to ambient water. In particular, the first peak position of the radial distribution function for supercooled water is $2.7 \AA$, slightly but noticeably smaller than the corresponding distance for ambient water equal to $2.76 \AA$ (see inset in Fig. 4).

This reduction of the oxygen-oxygen distance in supercooled water not only confirms the delocalized character of the proton as discussed above but also opens up to a likely correlation with the water density. Thus, the excess of $\left\langle E_{k}\right\rangle$ shown in Fig. 2 could be then considered as a microscopic counterpart of such a macroscopic quantity. The limited number of data points does not allow us to be more than qualitative here, but the polynomial fit shown in Fig. 2 (dotted line) is at least consistent with a maximum of $\left\langle E_{k}\right\rangle$ in the vicinity of water's density maximum. This latter anomaly is a terrific expression of the central role played by hydrogen bonding in determining the property of water [30]: the maximum of $\left\langle E_{k}\right\rangle$ could then be thought as the result of the balance between the weakening of the covalent bond (corresponding to a more delocalized proton) and the increasingly organized and open four-coordinated network of hydrogen bonds in water upon decreasing temperature. The possible link between the water density and the proton $\left\langle E_{k}\right\rangle$ observed in this Letter should stimulate theoretical work that explicitly includes the reduction of nearest neighbor water oxygens and the resulting delocalized character of the proton motion.
This work was supported within the CNR-CCLRC Agreement No. 01/9001 concerning collaboration in scientific research at the spallation neutron source ISIS. The financial support of the Consiglio Nazionale delle Ricerche in this research is hereby acknowledged.

*Author to whom correspondence should be addressed. bruni@fis.uniroma3.it

[1] P. G. Debenedetti, J. Phys. Condens. Matter 15, R1669 (2003).

[2] D. Eisenberg and W. Kauzmann, The Structure and Properties of Water (Oxford University Press, Oxford, 1969).

[3] O. Mishima and H. E. Stanley, Nature (London) 396, 329 (1998).

[4] P. L. Geissler et al., Science 291, 2121 (2001).

[5] F. N. Keutsch and R. J. Saykally, Proc. Natl. Acad. Sci. U.S.A. 98, 10533 (2001).

[6] G. F. Reiter, J. Mayers, and J. J. Noreland, Phys. Rev. B 65, 104305 (2002).

[7] C. Andreani et al., Adv. Phys. 54, 377 (2005).

[8] G.F. Reiter and R. Silver, Phys. Rev. Lett. 54, 1047 (1985).

[9] G. I. Watson, J. Phys. Condens. Matter 8, 5955 (1996).

[10] J. A. Morrone et al., J. Chem. Phys. 126, 234504 (2007).

[11] Momentum Distributions, edited by R. N. Silver and P.E. Sokol (Plenum Press, New York, 1989).

[12] C. Andreani et al., J. Chem. Phys. 115, 11243 (2001).

[13] G. F. Reiter et al., Braz. J. Phys. 34, 142 (2004).

[14] A. Pietropaolo et al., JINST 1, P04001 (2006).

[15] R. Senesi et al., Phys. Rev. Lett. 98, 138102 (2007).

[16] V. Garbuio et al., J. Chem. Phys. 127, 154501 (2007).

[17] C. Andreani et al., Nucl. Instrum. Methods Phys. Res., Sect. A 497, 535 (2003).

[18] C. A. Chatzimidriou-Dreisman et al., Phys. Rev. Lett. 79, 2839 (1997).

[19] R. Senesi et al., Phys. Rev. B 72, 054119 (2005).

[20] G. Reiter and P. Platzman, Phys. Rev. B 71, 054107 (2005).

[21] A. C. Evans et al., Phys. Rev. B 53, 3023 (1996).

[22] W. Götze, in Liquids, Freezing, and Glass Transition, edited by J. P. Hansen, D. Levesque, and J. Zinn-Justin (North-Holland, Amsterdam, 1991), p. 287.

[23] R. J. Speedy and C. A. Angell, J. Chem. Phys. 65, 851 (1976).

[24] L. Xu et al., Proc. Natl. Acad. Sci. U.S.A. 102, 16558 (2005).

[25] C. Pantalei et al. (private communication).

[26] G. F. Reiter et al., Phys. Rev. Lett. 97, 247801 (2006).

[27] G. F. Reiter, J. Mayers, and P. Platzman, Phys. Rev. Lett. 89, 135505 (2002).

[28] H. J. Bakker and H. K. Nienhuys, Science 297, 587 (2002).

[29] A. Botti et al., J. Chem. Phys. 117, 6196 (2002).

[30] D. Liu et al., Proc. Natl. Acad. Sci. U.S.A. 104, 9570 (2007). 